\title{
Molecular Characterization of Theileria annulata Isolates from Northern India Based on Tams1 Gene
}

\author{
M. R. Arun Raj, Stuti Vatsya* and Rajeev Ranjan Kumar
}

Department of Veterinary Parasitology, College of Veterinary and Animal Sciences, G.B. Pant University of Agriculture and Technology, Pantnagar, Uttarakhand, 263145 India

*Corresponding author

\section{A B S T R A C T}

\begin{tabular}{|l|}
\hline Ke y w o r d s \\
$\begin{array}{l}\text { Theileria annulata, } \\
\text { Tamsl gene, Genetic } \\
\text { diversity, Phylogenetic } \\
\text { analysis }\end{array}$ \\
\hline Article Info \\
\hline $\begin{array}{l}\text { Accepted: } \\
\text { 20 February } 2018 \\
\text { Available Online: } \\
\text { 10 March } 2018\end{array}$ \\
\hline
\end{tabular}

Considering increasing reports of bovine tropical theileriosis from various parts of northern India and the impact of this disease to sustainable livestock production, the present study was carried out with the objective of understanding the genetic characterization of Theileria annulata based on Tams1 gene sequence analysis. Six different isolates of $T$. annulata from four districts of northern India namely Nainital (two isolates- Nainital_31 and Nainital_255), UdhamSinghNagar (two isolatesUdhamSinghNagar_1 and UdhamSinghNagar_314) and one isolate each from Dehradun, and Pithoragarh were included in the study. The sequence analysis of the partial Tams 1 gene obtained from different regions selected for the study revealed high level of diversity between regions and within the region. The partial Tams1 gene sequences of different isolates when subjected to phylogenetic analysis revealed that the UdhamSinghNagar_314 and Pithoragarh isolates originated from similar ancestor whereas Dehradun and UdahmSinghNagar_1 had a different ancestral lineage.

\section{Introduction}

Haemoprotozoan parasites like Babesia, Theileria and Trypanosoma often present a challenge to successful livestock farming. Bovine tropical theileriosis (BTT) caused by Theileria annulata occurs in different parts of India (Acharya et al., 2017, Sudan et al., 2017) including sub-Himalyan region of northern India (Rialch et al., 2013, Kohli et al., 2014a). In India, the disease affects imported high grade/ cross-bred cattle and young indigenous calves (Acharya et al., 2017) in which theileriosis is a severe and often lethal disease. These factors impose serious constraints upon livestock production. In India, around 10 million cattle are at risk for tropical theileriosis with an annual economic loss of US \$ 384.3 million per annum (Minjauw and Mcleod, 2003). Round the year, prevalence of infective ticks in the surroundings and transplacental transmission of $T$. annulata from dam to foetus cause per acute and fatal disease, especially in the semiarid regions of India (Godara et al., 2009).

Molecular diagnostic based assays have been developed which have proven to be effective in detecting theilerial infection in carrier animals. PCR based assay for species-specific 
molecular diagnosis is considered to be superior in terms of the sensitivity and specificity to microscopic methods (Mosqueda et al., 2012). Genes which are normally being targeted for species specific detection of $T$. annulata are, srRNA gene (Georges et al., et al., 2001), Tamsl gene (D'Oliveria et al., 1995), Heat shock protein 70 (HSP70) gene (Shayan et al., 1998), $\beta$ tubulin gene (Caccio et al., 2000) and Cytochrome $\mathrm{b}$ gene (Bilgic et al., 2010). However, the gene coding for an immunodominant major merozoite/piroplasm surface antigen of $T$. annulata (Tams 1 ) is commonly used for PCR based assay.

Considering increasing reports of bovine tropical theileriosis from various parts of northern India and the impact of this disease to sustainable livestock production, the present study was carried out with the objective of understanding the genetic characterization of T. annulata based on Tams l gene sequence analysis.

\section{Materials and Methods}

\section{Sample collection and DNA isolation from blood}

Blood samples (1 $\mathrm{ml}$ aliquot) were collected in clean sterile vacutainer, coated with EDTA anticoagulant, from the jugular vein of earlier confirmed cross-bred cows (through microscopic observation of blood smears). Six different isolates of Theileria annulata from four districts of northern India namely Nainital (two isolates- Nainital_31 and Nainital_255), UdhamSinghNagar (two isolates- Udham SinghNagar_1 and UdhamSinghNagar_314) and one isolate each from Dehradun, and Pithoragarh were included in the study. District Udham Singh Nagar (U.S. Nagar) $\left(28.97^{\circ} \mathrm{N}\right.$ and $79^{\circ} .41^{\prime} \mathrm{E}$; $550 \mathrm{mt}$ above sea level) and Dehradun (30.318' N and $78^{\circ} .029^{\prime} \mathrm{E}$; $435 \mathrm{mt}$ above sea level) constituted the tarai zone while two districts namely
Nainital $\left(29^{\circ} .23^{\prime} \mathrm{N}\right.$ and $79^{\circ} .27^{\prime} \mathrm{E} ; 1938 \mathrm{mt}$ above sea level) and Pithoragarh $\left(30.00^{\circ} .58^{\prime} \mathrm{N}\right.$ and $80^{\circ} .33^{\prime} \mathrm{E} ; 1650 \mathrm{mt}$ from sea level) constituted the hill zone.

\section{DNA extraction}

DNA from whole blood sample was extracted using modified protocol of Sambrook et al., (1989). Briefly, $300 \mu \mathrm{l}$ of whole blood sample along with $700 \mu \mathrm{l}$ of lysis buffer (1 M Tris-Cl, $5 \mathrm{M} \mathrm{NaCl}, 0.5 \mathrm{M}$ EDTA, 10\% SDS), $5 \mu \mathrm{l}$ Proteinase K (Genei, Banglore. 20mg/ml) and $2 \mu \mathrm{l}$ of RNase $\mathrm{A}$ were added and mixed thoroughly in a micro-centrifuge tube of $2 \mathrm{ml}$ capacity by vortexing to form a homogenous mixture. This homogenous mixture was incubated overnight at $56^{\circ} \mathrm{C}$ in a shaker incubator (New Brunswick Eppendorf, Germany). To the lysed mixture, Tris saturated phenol was added in equal volume $(1 \mathrm{ml})$ and mixed thoroughly by vortexing. Mixture was then centrifuged at 2,000 rpm for 15 minutes. Supernatant was transferred to a fresh sterile micro-centrifuge tube of $2 \mathrm{ml}$ capacity and equal volume of phenol: chloroform: isoamylalcohol (25:24:1) was added and mixed thoroughly by vortexing. The mixture was then centrifuged at 12,000 rpm for 10 minutes. The upper aqueous phase was transferred to a new sterile microcentrifuge tube of $2 \mathrm{ml}$ capacity and to it was added equal volume of chloroform: isoamylalcohol (24:1) and mixed thoroughly by vortexing. The mixture was centrifuged at $12,000 \mathrm{rpm}$ for 10 minutes. Upper aqueous phase was transferred to a new sterile microcentrifuge tube of $2 \mathrm{ml}$ capacity. Chilled isopropyl alcohol (double the volume) was added to the aqueous phase to precipitate the DNA followed by centrifugation at 10,000 rpm for 1 minute. The DNA pellet thus obtained was washed twice with $70 \%$ chilled molecular grade ethanol. DNA pellet was air dried by keeping at $37^{\circ} \mathrm{C}$ for few minutes and finally $50 \mu 1$ nuclease free water was added to 
resuspend the DNA. The extracted DNA was analysed using agarose gel electrophoresis (Figure 1).

\section{Allele-specific polymerase chain reaction (AS-PCR) assay}

Amplification of Tams1 gene of Theileria annulata

Tams1 gene was amplified as per the method described by Kirvar et al., (2000) using the following oligonucleotide primers:

Forward primer:

5' - ATGCTGCAAATGAGGAT-3'

Reverse primer:

5' - GGACTGATGAGAAGACGATGAG-3'

PCR was carried out in thin walled $0.2 \mathrm{ml}$ PCR tubes in $25 \mu 1$ reaction volume. Genomic DNA isolated from various blood samples were used as templates. The PCR mixture consisted of $5 \mu 1$ of DNA template, $2.5 \mu 1$ of $10 \mathrm{X}$ PCR buffer (without $\mathrm{MgCl}_{2}$ ), $1.5 \mu \mathrm{l}$ of $25 \mathrm{mM}$ $\mathrm{MgCl}_{2}, \quad 0.2 \mathrm{mM}$ of each dNTP (Genei, Banglore), 10 pmol of each primer (forward and reverse) and $1 \mathrm{U}$ of $\mathrm{Taq}$ Polymerase (Genei, Banglore).

The volume of reaction mixture was made up to $25 \mu \mathrm{l}$ by nuclease free water. PCR was performed in an automated EP-Gradient $S$ thermocycler (Eppendorf, Germany) programmed for the following conditions:

The cycling conditions consisted of 1 cycle of initial denaturation at $94^{\circ} \mathrm{C}$ for 5 min followed by 35 cycles, each consisting of denaturation at $94^{\circ} \mathrm{C}$ for $1 \mathrm{~min}$, annealing at $60^{\circ} \mathrm{C}$ for $1 \mathrm{~min}$ and elongation at $72{ }^{\circ} \mathrm{C}$ for $1 \mathrm{~min}$. followed by a final extension step at $72^{\circ} \mathrm{C}$ for $10 \mathrm{~min}$. The PCR product was analysed using agarose gel electrophoresis. The rest of the PCR product was kept at $-20^{\circ} \mathrm{C}$ until further use.

\section{Nucleotide sequencing}

The PCR products of Tamsl gene amplification from blood samples of different agro climatic areas were sent to Sci Genom Labs Pvt. Ltd., Cochin, Kerala for sequencing (double pass analysis using forward and reverse primer). $100 \mu \mathrm{l}$ of PCR product was purified using Himedia PCR purification kit. Purification was done as described by the manufacturer. Sequence analysis was done using an automated ABI 3100 Genetic Analyser (Applied Biosystems, Germany) which uses ABI's AmpliTaq FS dye terminator cycle sequencing chemistry which is based on Sanger's sequencing method.

Correct sequences for all the PCR products were determined individually from the chromatogram and sequence data obtained using both forward and reverse primers. Alignments of the sequence of different regions were done with the programme BioEdit available in Mega 5 software. The sequenced partial Tams 1 gene from different agro climatic regions were compared and analysed with that of already published gene sequence available in the Gene Bank. After alignment of these genes, their nucleotide variability and amino acid variability were studied.

\section{Phylogenetic analysis}

The nucleotide sequences were compared and phylogenetic analysis was done using the sequence data available in GenBank and the sequence data obtained from the present study. The sequence data was aligned using the software Mega 5 with Clustal $W$ multiple sequence alignment.

Percent similarity/percent divergence and phylogenetic trees were derived by using BioEdit programme available in Mega 5 software. 


\section{Results and Discussion}

Amplification of partial Tams1 gene using AS-PCR assay yielded a gene of length 785bp in all cases (Figure 2).

\section{Sequence analysis of Tams 1 gene}

The Tamsl gene sequences received were compared with available sequence of Tams 1 gene in GenBank using the nucleotide BLAST analysis available in the NCBI website (www.ncbi.nlm.nih.gov).

Partial Tams1 gene sequence obtained from Dehradun isolate showed $98 \%$ identitiy with 08\#05 Isolate and 93\% with Tamil Nadu and tH4 isolate. Pithoragarh isolate exhibited $96 \%$ identitiy with $80 \# 05$ isolate and $95 \%$ with Tamil Nadu and tH4 isolate. Two isolates from UdhamSinghNagar were studied namely, UdhamSinghNagar_1 (Accession number KM061795) and UdhamSinghNagar_314 (Accession number KM061796.1). UdhamSinghNagar_1 showed $92 \%$ and $93 \%$ identity with tH4 and 08\#05 isolates, respectively.

Whereas UdhamSinghNagar_314 had 95\% and $94 \%$ identity with tH4 and $08 \# 05$ isolates, respectively. In Nainital district two isolates were sequenced namely Nainital_31 (Accession number KM061797.1) and Nainital_255 (Accession number KM061798.2) showing an identity of $94 \%$ and $95 \%$ with tH4 isolate, respectively. Nainital_31 showed 97\% and 93\% identity with 08\#05 isolate and Tamil Nadu isolate respectively.

The nucleotide sequences of different isolates of Tams1 genes of present study and Hissar strain (Accession number AF214843.1) sequences were translated to amino acid sequences for comparison. The sequence analysis of the partial Tams1 gene obtained from different regions selected for the study revealed high level of polymorphism between regions and within the region. In Dehradun isolate (Accession number KM061794.1) the nucleotide variations in the Tams1 gene sequence as compared to Hissar strain resulted in change in the translated amino acid sequence at 23 sites. These changes are in correspondence with the change in nucleotide sequence. At position 41(Leucine instead of Threonine), position 42 (Alanine instead of Leucine), position 49 (Valine instead of Threonine), position 50 (Phenylalanine instead of Serine), position 51 (Histidine instead of Lycine) position 52 (Threonine instead of Aspargine), position 53 (Arginine instead of Valine), position 55 (Serine instead of Proline), position 61 (Leucine instead of Threonine), position 103 (Lysine instead of Arginine), position 108 (Proline instead of Alanine), position 152 (Alanine instead of Proline), position 171 (Glutamate instead of Aspartate), position 178 (Threonine instead of Leucine), position 180 and 183 (Aspartate instead of Glutamate), position 187 (Alanine instead of Proline), position 188 (Aspartate instead of Aspargine), position 189 (Lycine instead of Aspargine), position 194 (Glutamate instead of Aspartate), position 198 (Tyrosine instead of Phenylalanine), position 237 (Serine instead of Alanine), and position 252 (Arginine instead of Histidine).

In two Nainital isolates, Nainital_31 and Nainital_255 amino acids were observed to be changed at 10 positions. This change in amino acid were not at same sites in both the isolates i.e. changes in six positions were same but the rest four sites were different. Six sites at which same changes were found were at position 125 (Phenylalanine instead of Valine), position 152 (Alanine instead of Proline), position 171 (Glutamate instead of Aspartate), position 178 (Threonine instead of Leucine), position 180 and 183 (Aspartate instead of Glutamate). 
Fig.1 Purified genomic DNA

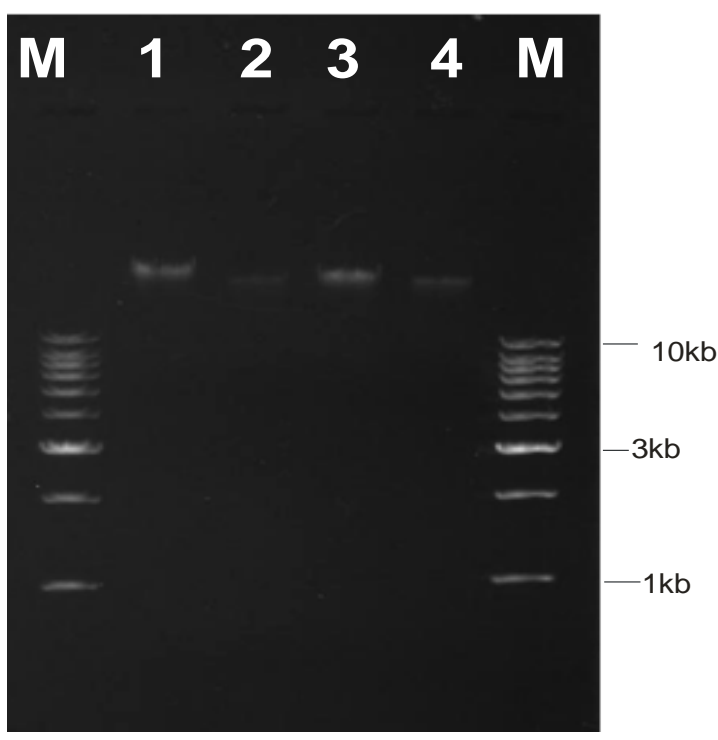

Lane M: $1 \mathrm{~kb}$ DNA Ladder Lane 1: Positive Lane 2: Positive Lane 3: Positive Lane 4: Negative

Fig.2 Amplified partial Tams1 gene

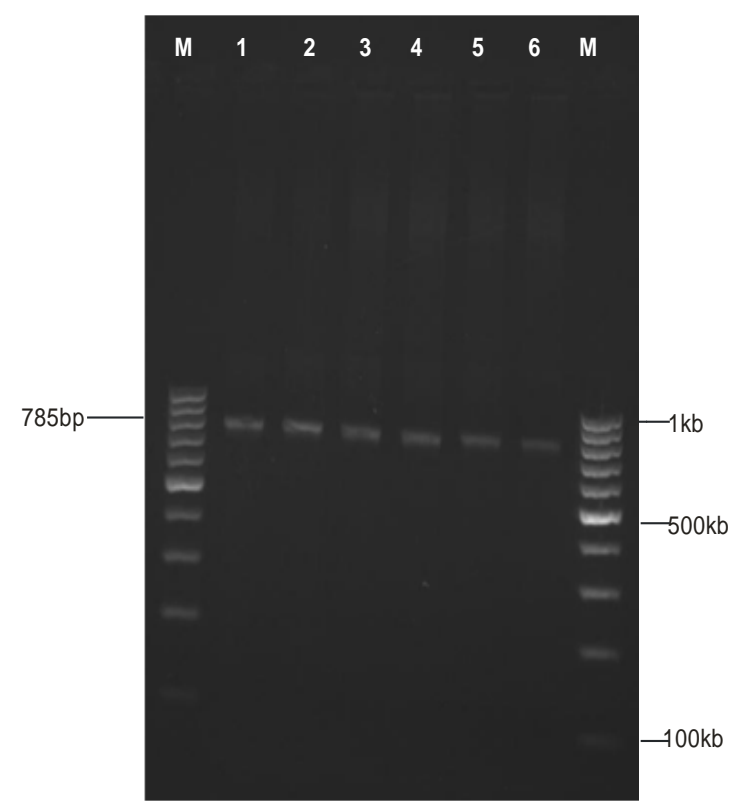

Lane M: 100 bp DNA Ladder Lane 1: U S Nagar Lane 2: U S Nagar Lane 3: Dehradun Lane 4: Pithoragarh

Lane 5: Nainital

Lane 6: Nainital 
Fig.3 Phylogenetic tree based on a comparison of partial Tams1 isolates obtained from different agro-climatic zones of Uttarakhand to other GeneBank sequences.

The lineages are shown on the tree

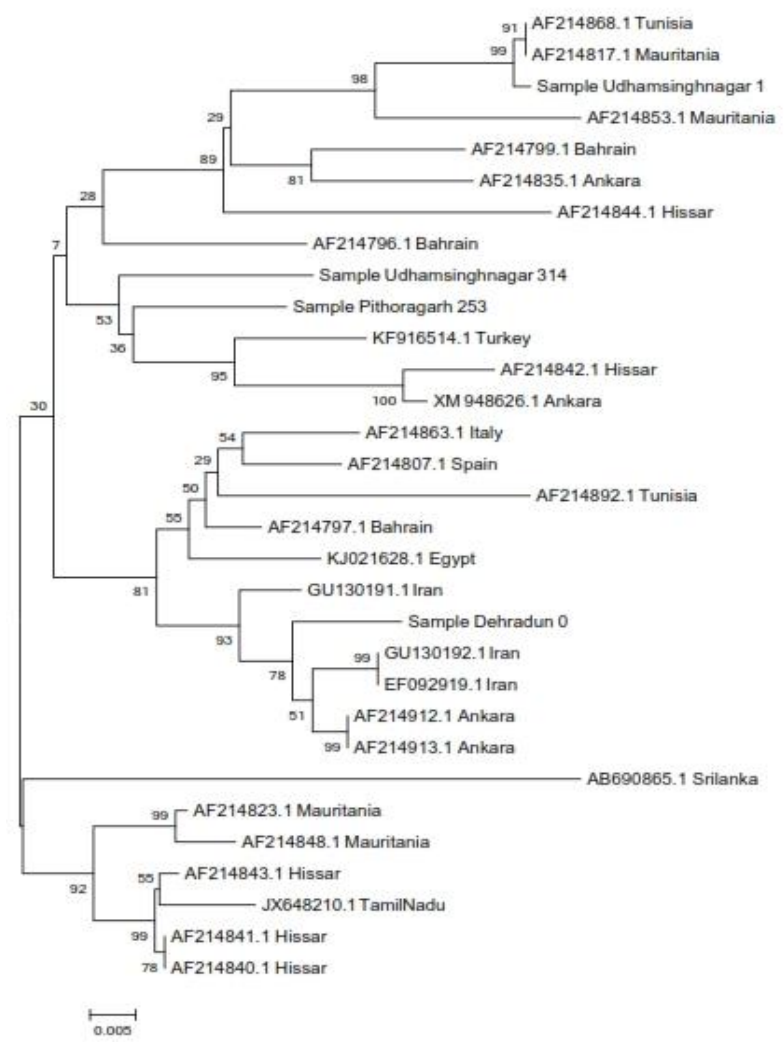

Fig.4 Percent identity and divergence between six isolates and ten published sequences of Tamsl gene

\begin{tabular}{|c|c|c|c|c|c|c|c|c|c|c|c|c|c|c|c|c|c|}
\hline \multicolumn{18}{|c|}{ Percent Identity } \\
\hline & 1 & 2 & 3 & 4 & 5 & 6 & 7 & 8 & 9 & 10 & 11 & 12 & 13 & 14 & 15 & 16 & \\
\hline 1 & & 84.2 & 82.7 & 84.4 & 85.6 & 84.5 & 83.6 & 87.8 & 82.5 & 88.7 & 86.5 & 61.0 & 61.5 & 87.3 & 83.7 & 89.1 & 1 \\
\hline 2 & 8.0 & & 92.7 & 94.0 & 92.5 & 94.1 & 95.0 & 81.5 & 75.5 & 81.1 & 80.1 & 54.9 & 55.4 & 81.0 & 77.2 & 84.3 & 2 \\
\hline 3 & 9.9 & 7.0 & & 90.0 & 93.1 & 90.2 & 96.3 & 82.4 & 76.3 & 82.5 & 80.7 & 53.3 & 52.7 & 82.7 & 77.9 & 81.0 & 3 \\
\hline 4 & 9.6 & 5.0 & 8.5 & & 92.9 & 99.9 & 92.8 & 82.7 & 75.9 & 81.1 & 80.2 & 57.1 & 55.1 & 80.7 & 78.2 & 90.0 & 4 \\
\hline 5 & 8.0 & 6.7 & 5.1 & 7.7 & & 92.8 & 93.3 & 82.3 & 81.9 & 85.2 & 80.1 & 55.5 & 54.5 & 84.2 & 80.3 & 82.8 & 5 \\
\hline 6 & \begin{tabular}{|l}
9.5 \\
\end{tabular} & 4.8 & 8.4 & 0.1 & 7.9 & & 92.9 & \begin{tabular}{|l}
82.7 \\
\end{tabular} & \begin{tabular}{|l}
75.9 \\
\end{tabular} & 81.1 & 80.2 & 57.1 & 55.1 & \begin{tabular}{|l|l}
80.7 \\
\end{tabular} & \begin{tabular}{|l}
78.2 \\
\end{tabular} & 90.1 & 6 \\
\hline 7 & 8.7 & 5.3 & 3.0 & 6.3 & 5.8 & 6.1 & & 85.4 & 76.3 & 81.1 & 82.8 & 55.1 & 54.2 & 82.3 & 77.5 & 83.1 & 7 \\
\hline 8 & \begin{tabular}{|l}
8.5 \\
\end{tabular} & 5.9 & 4.6 & 6.2 & 6.6 & 6.2 & 1.0 & & 88.8 & 90.6 & 97.5 & 69.9 & 70.0 & 94.0 & 91.5 & 91.5 & 8 \\
\hline 9 & \begin{tabular}{|l|l}
10.0 \\
\end{tabular} & 8.8 & 6.5 & 10.1 & 1.9 & 10.1 & 7.6 & 8.3 & & 87.9 & 89.8 & 72.6 & 72.7 & \begin{tabular}{|l}
89.2 \\
\end{tabular} & 92.8 & 84.3 & 9 \\
\hline 10 & 9.9 & 8.6 & 6.9 & 10.5 & 5.4 & 10.5 & 8.6 & 9.0 & 6.7 & & 89.5 & 64.5 & 65.1 & 94.7 & 91.0 & 90.0 & 10 \\
\hline 11 & 8.8 & 5.6 & 4.8 & 7.4 & 7.5 & 7.4 & 2.1 & 1.7 & \begin{tabular}{|l}
8.1 \\
\end{tabular} & 9.1 & & 70.3 & 71.1 & 92.7 & \begin{tabular}{|l}
92.1 \\
\end{tabular} & 89.5 & 11 \\
\hline 12 & \begin{tabular}{|l|}
8.3 \\
\end{tabular} & 3.2 & 6.3 & 1.8 & 4.8 & 1.8 & 2.7 & 1.8 & \begin{tabular}{|l|}
6.0 \\
\end{tabular} & 7.8 & 3.2 & & 96.2 & 66.3 & 70.8 & 66.4 & 12 \\
\hline 13 & \begin{tabular}{|l|}
7.5 \\
\end{tabular} & 1.0 & 6.1 & 4.5 & 5.8 & 4.5 & 3.2 & 2.5 & \begin{tabular}{|l|l}
7.4 \\
\end{tabular} & 7.2 & 2.5 & 2.6 & & 67.0 & 71.8 & 65.3 & 13 \\
\hline 14 & 10.0 & 6.9 & 4.7 & 9.1 & 4.7 & 9.1 & 5.1 & 5.4 & 5.5 & \begin{tabular}{|l|}
4.7 \\
\end{tabular} & 5.6 & 5.6 & 5.0 & & 92.3 & 90.0 & 14 \\
\hline 15 & 10.2 & 7.1 & 6.1 & 7.7 & 4.9 & 7.7 & 6.6 & 7.1 & 6.5 & 4.7 & 7.4 & 5.3 & 5.0 & \begin{tabular}{|l|}
4.1 \\
\end{tabular} & & 87.2 & 15 \\
\hline 16 & \begin{tabular}{|l|l}
10.0 \\
\end{tabular} & 5.7 & 9.8 & 0.4 & 9.0 & 0.3 & 7.2 & 6.2 & \begin{tabular}{|l}
9.9 \\
\end{tabular} & 10.9 & 7.4 & \begin{tabular}{|l|}
1.8 \\
\end{tabular} & 4.5 & 9.1 & \begin{tabular}{|l}
8.1 \\
\end{tabular} & & 16 \\
\hline & 1 & 2 & 3 & 4 & 5 & 6 & 7 & 8 & 9 & 10 & 11 & 12 & 13 & 14 & 15 & 16 & \\
\hline
\end{tabular}

AB690865.1_Srilanka.seq AF214799.1_Bahrain.seq AF214807.1_Spain.seq AF214817.1_Mauritania.se AF214841.1_Hissar.seq AF214868.1_Tunisia.seq AF214912.1_Ankara.seq EF092919.1_Iran.seq JX648210.1_TamilNadu.se KF916514.1_Turkey.seq 2.SampleDehradun_1.seq 3.SampleNainital_255.seq 4.SampleNainital_31.seq 5.SamplePithoragarh_253. 6.SampleUS Nagar_314.se 7.SampleUS Nagar_1.seq 
Fig.5 Phylogenetic tree based on a comparison of partial Tams1 isolates obtained from Nainital district of Uttarakhand to other GeneBank sequences. The lineages are shown on the tree

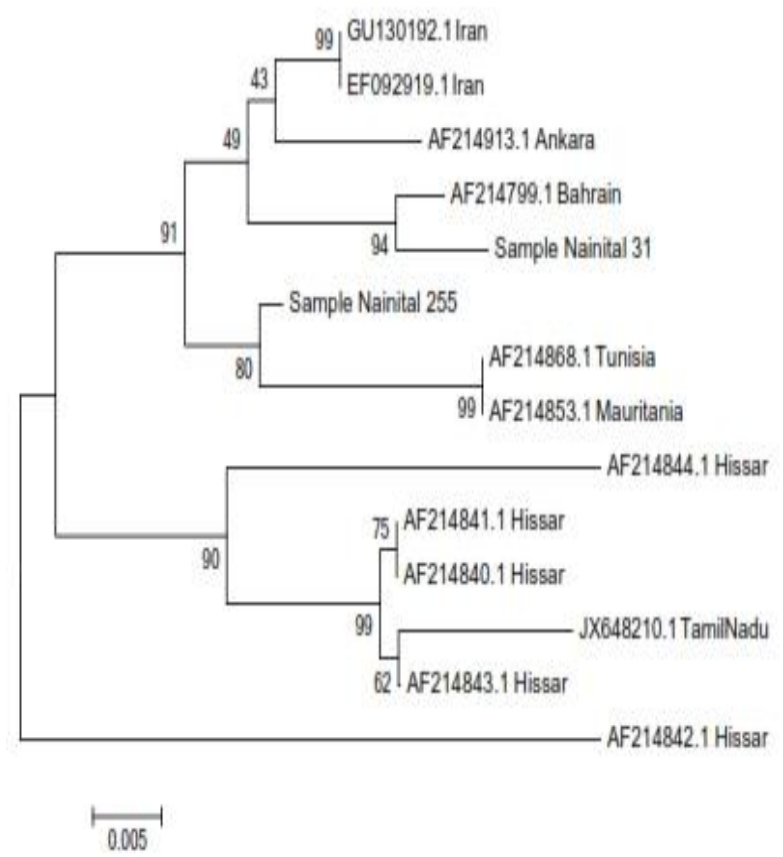

In case of Nainital_31 isolate other than these six common changes, four more changes were noted i.e. 164 position (Arginine instead of Lycine), position 187 (Alanine instead of Proline), position 188 (Aspartate instead of Asparagine) and 189 position (Lysine instead of Asparagine). Likewise in Nainital_255 at 153 and 237 position it was Alanine instead of serine, 251 position it was Valine, $252^{\text {nd }}$ position it was a stop codon instead of Histidine.

In Pithoragarh isolate (Accession number KM061799) amino acids were found to be changed at 14 locations, position 50 (Leucine instead of Serine), position 51 (Asparagine instead of Lysine), position 52 (Threonine instead of Asparagine), position 103 (Lysine instead of Arginine), position 108 (Proline instead of Alanine), position 152 (Threonine instead of Proline), position 164 (Arginine instead of Lysine), position 180 (Aspartate instead of Glutamate), position 194 (Glutamate instead of Aspartate), position 201
(Serine instead of Alanine), position 206 (Phenylalanine instead of Valine), position 208 (Lycine instead of Arginine), position 210 (Tyrosine instead of Aspartate) and position 245 (Leucine instead of Arginine).

In UdhamSinghNagar isolates, US Nagar_1 and US Nagar_314, it was found that amino acids sequences were changed in 31 and 20 positions, respectively. Of these changes 7 were at same sites, position 50 (Serine instead of Phenylalanine), position 51(Aspartate instead of Lycine), position 52 (Threonine instead of Asparagine), position 53 (Arginine instead of Valine), position 103 (Lysine instead of Arginine), position 108 (Proline instead of Alanine) and position 152 (Alanine instead of Proline).

In US Nagar_1 there were 24 amino acid changes other than above mentioned ones; they were at positions 42 (Alanine instead of Leucine), 27 (Leucine instead of Glutamate), 28 (Argenine instead of Lysine), 57 (Alanine 
instead of Threonine), 58 (Valine instead of Threonine), 59 (Valine instead of Glutamate), 90 (Lysine instead of Glutamine), 92 (Valine instead of Phenylalanine), 105 (Leucine instead of Asparagine), 125 (Serine instead of Valine), 153 (Serine instead of Alanine), 159 (Threonine instead of Alanine), 162 (Serine instead of Threonine), 170 (Asparagine instead of Lysine) 171 (Glutamate instead of Aspartate), 178 (Threonine instead of Leucine), 180 and 183 (Aspartate instead of Glutamate), 198 (Tyrosine instead of Phenylalanine), 237 (Serine instead of Alanine), 252 (Tryptophan instead of Histidine) and 256 (Lysine).

In Nainital_255 there were 13 sites showing amino acid changes other than the common changes among the isolates. They were position 57 (Serine instead of Threonine), 63 (Alanine instead of Lysine), 105 (Aspartate instead of Asparagine), 125 (Phenylalanine instead of Valine), 157 (Serine instead of Proline), 164 (Argenine instead of Lysine), 194 (Glutamate instead of Aspartate), 201 (Serine instead of Alanine), 210 (Tyrosine instead of Aspartate), 245 (Leucine instead of Aspargine), 247 (Glutamate instead of Glycine), 250 (Glutamate instead of Asparagine) and 252 (Proline instead of Histidine). Tams1 polypeptide is an immunodominant major merozoite piroplasm surface antigen of $T$. annulata which could be used as a marker for epidemiological studies and phylogenetic analysis (Wang et al., 2014). The Tamsl-encoding gene has been developed for PCR-based assays to detect $T$. annulata infections in bovines.

\section{Phylogenetic analysis of the partial Tams1 gene obtained from different agro-climatic zones of Uttarakhand}

The partial Tams1 gene sequences of different isolates (Dehradun, Pithoragarh, and UdhamSinghNagar) in this study were subjected to phylogenetic analysis with 26 GeneBank sequences. The sequences were aligned by Clustal W multiple alignment program and the phylogenetic tree was made using neighbour-joining method with 2000 bootstrap in Mega 6 software. The result of phylogenetic analysis generated in phylogenetic tree ((Figure 3 ) points out origin and evolution of various isolates in this study. In case of Nainital isolates (Nainital_31 and Nainital_255), sequences were short (411bp and $449 \mathrm{bp}$ ) to form a phylogenetic tree with available GeneBank sequences. Twelve available GeneBank sequences were aligned with Nainital isolates and trimmed to approx 450bp with the help of Seqman program in DNASTAR 6 computer software. After this a phylogenetic tree was made as mentioned above ((Figure 4). Percent identity and divergence between six isolates and ten published sequences of Tamsl gene was made with Megalign program in DNASTAR 6 computer software (Figure 5).

The phylogenetic tree revealed that the UdhamSinghNagar_314 and Pithoragarh isolates originated from similar ancestor whereas Dehradun and UdahmSinghNagar_1 had a different ancestral lineage. Genetic analysis of parasite populations can provide important information about the epidemiology of disease and may facilitate the development of rational control approaches (Al-Hamidi et al., 2016). Tams1 gene of T. annulata is antigenically diverse (Dickson and Shiels, 1993) and T. annulata may generate novel antigenic Tamsl types by differential glycosylation (Katzer et al., 1998). The high diversity of antigen genes Tamsl in $T$. annulata confers a selective advantage to parasite genotypes by facilitating evasion from a protective immune response (Wang et al., 2014) thus promoting a more widespread distribution and survival of this species. The phylogenetic variability and molecular genetic characterization of Tams1 will help in 
understanding the relationship between the molecular evolutionary history of $T$. annulata and the emergence and spread of new ones in the area under study.

\section{Acknowledgements}

The authors are thankful to the Director, Experiment Station and Dean, College of Veterinary and Animal Science, G.B. Pant University of Agriculture and Technology, Pantnagar for the facilities provided during the course of study.

\section{References}

Acharya, A.P., Panda, S.K., Prusty, B.K. 2017. Diagnosis and confirmation of Theileria annulata infection in cattle in Odisha. Indian Journal of Entomology and Zoology Studies 5: 1543-1546.

Al-Hamidi, S., Weir, W., Kinnaird, J.m Tageledin, M., Beja-Pereira, A., Morrison, I., Thompson, J., Tait, A., Shiels, B., Babiker, H.A. 2016. Theileria lestoquardi displays reduced genetic diversity relative to sympatric Theileria annulata in Oman. Infection, Genetics and Evolution. 43: 297-306.

Bilgic, H.B., Karagenc, T., Shiels, B., Tait, A., Eren, H., Weir, W. 2010. Evaluation of cytochrome $\mathrm{b}$ as a sensitive target for PCR-based detection of Theileria. annulata carrier animals. Vet. Parasitol. 174: 341-347.

Caccio, S., Camma, C., Onuma, M., Severini, C. 2000. The beta-tubulin gene of Babesia and Theileria parasites is an informative marker for species discrimination. Int. J. Parasitol. 30: 1181-1185.

D’Oliveira, C., Vandermerve, M., Habela, M., Jacquiet, P., Jongejan, F. 1995. Detection of Theileria annulata in blood samples of carrier cattle by PCR. J. Clin. Microbiol. 33: 2665-2669.

Dickson, J., Shiels, B.R. 1993. Antigenic diversity of a major merozoite surface molecule in Theileria annulata. Mol. Biochem. Parasitol. 57: 55-64.

Georges, K., Loria, G.R., Riili, S., Greco, A., Caracappa, S., Jongejan, F., Sparagano, O. 2001. Detection of haemoparasites in cattle by reverse line blot hybridisation with a note on the distribution of ticks in Sicily. Vet. Parasitol. 99: 273-286.

Godara, R., Sharma, R.L., Sharma, C.S. 2009. Bovine tropical theileriosis in a neonate calf. Trop. Anim. Hlth. Prodn. 42: 551553.

Katzer, F., McKellar, S., Ben Miled, L., d'Oliveira, C., Shiels, B. 1998. Selection for antigenic diversity of Tams1, the major merozoite antigen of Theileria annulata. Ann N Y Acad Sci. 849: 96108.

Kirvar, E., Ilhan T., Katzer, F., HooshmandRad, P., Zweygarth, E., Gerstenberg, C., Phipps, P., Brown, C.G.D. 2000. Detection of Theileria annulata in cattle and vector ticks by PCR using the Tams1 gene sequences. Parasitol. 120: 245-254.

Kohli, S., Atheya, U.K., Srivastava, S.K., Banerjee, P.S., Garg, R. 2014a. Outbreak of theileriosis and anaplasmosis in herd of Holstein crossbred cows of Dehradun district of Uttarakhand, India: A Himalyan region. International J. Livestock Prod. 5: 182185.

Minjauw, B., McLeod, A. 2003. Tick- borne diseases and poverty. The impact of ticks and tick-borne diseases on the livelihood and marginal livestock owners in India and Eastern and Southern Africa. Research report, DFID Animal Health Programme, Centre of Tropical Veterinary Medicine, University of Edinburgh. pp. 1-124. 
Mosqueda, J., Olvera-Ramirez, A., AquilarTipacamu, G., Canto, G.J. 2012. Current advances in detection and treatment of babesiosis. Curr. Med. Chem. 19, 1504-1518.

Rialch, A., Vatsya, S., Kumar, R. R. 2013. Prevalence of some blood parasites in large ruminants of Tarai region of Uttarakhand. Pantnagar J. Res. 11: 315316.

Sambrook, J., Fritsch, E. F., Maniatis, T. 1989. Molecular Cloning: A Laboratory Manual. Vol 2. $2^{\text {nd }}$ ed. New York, Cold Spring Harbor Laboratory Press. pp. 7.26-7.45.

Shayan, P., Biermann, R., Schein, E., Gerdes, J., Ahmed, J.S. 1998. Detection and differentiation of Theileria annulata and
Theileria parva using macroschizontderived DNA probes. Ann. N.Y. Acad. Sci. 849: 88-95.

Sudan, V., Shanker, D., Jaiswal, A., Singh, A., Pandey, V. 2017. Standardization and validation of simple PCR, duplex PCR and RAPD in comparison to blood smear examination for diagnosing bovine tropical theileriosis. Biologicals. 46: 88-91.

Wang, J., Yang, X., Wang, Y., Jing, Z., Meng, K., Liu, J., Guo, H., Xu, R., Cheng, Z. 2014. Genetic diversity and phylogenetic analysis of Tams 1 of Theileria annulata isolates from three continents between 2000 and 2012. Central European J. of Immunol. 39: 476-484.

\section{How to cite this article:}

Arun Raj, M.R., Stuti Vatsya and Rajeev Ranjan Kumar. 2018. Molecular Characterization of Theileria annulata Isolates from Northern India Based on Tamsl Gene. Int.J.Curr.Microbiol.App.Sci. 7(03): 2351-2360. doi: https://doi.org/10.20546/ijcmas.2018.703.276 